\title{
Positive autoregulation of GDNF levels in the ventral tegmental area mediates long-lasting inhibition of excessive alcohol consumption
}

\author{
S Barak ${ }^{1,2}$, S Ahmadiantehrani ${ }^{1,3}$, V Kharazia ${ }^{1}$ and D Ron ${ }^{1,2,3}$
}

Glial cell line-derived neurotrophic factor (GDNF) is an essential growth factor for the survival and maintenance of the midbrain dopaminergic (DA-ergic) neurons. Activation of the GDNF pathway in the ventral tegmental area (VTA), where the GDNF receptors are expressed, produces a long-lasting suppression of excessive alcohol consumption in rats. Previous studies conducted in the DA-ergic-like cells, SHSY5Y, revealed that GDNF positively regulates its own expression, leading to a longlasting activation of the GDNF signaling pathway. Here we determined whether GDNF activates a positive autoregulatory feedback loop in vivo within the VTA, and if so, whether this mechanism underlies the long-lasting suppressive effects of the growth factor on excessive alcohol consumption. We found that a single infusion of recombinant GDNF (rGDNF; $10 \mu \mathrm{g}$ ) into the VTA induces a long-lasting local increase in GDNF mRNA and protein levels, which depends upon de novo transcription and translation of the polypeptide. Importantly, we report that the GDNF-mediated positive autoregulatory feedback loop accounts for the long-lasting inhibitory actions of GDNF in the VTA on excessive alcohol consumption. Specifically, the long-lasting suppressive effects of a single rGDNF infusion into the VTA on excessive alcohol consumption were prevented when protein synthesis was inhibited, as well as when the upregulation of GDNF expression was prevented using short hairpin RNA to focally knock down GDNF mRNA in the VTA. Our results could have implications for the development of long-lasting treatments for disorders in which GDNF has a beneficial role, including drug addiction, chronic stress and Parkinson's disease.

Translational Psychiatry (2011) 1, e60; doi:10.1038/tp.2011.57; published online 13 December 2011

\section{Introduction}

Glial cell line-derived neurotrophic factor (GDNF) is a secreted growth factor that was initially identified in a glialderived cell line ${ }^{1}$ GDNF is expressed throughout the central nervous system during development, and high levels of GDNF mRNA are present in the adult neurons in brain regions such as the striatum, thalamus, cortex and hippocampus. ${ }^{2}$ In the mesolimbic/nigrostriatal systems, GDNF is produced in striatal neurons ${ }^{3,4}$ and is retrogradely transported by dopaminergic (DA-ergic) neurons to the substantia nigra ${ }^{5,6}$ and the ventral tegmental area (VTA), ${ }^{7}$ where the GDNF receptors, GFR $\alpha 1$ and Ret, are highly expressed. ${ }^{8-10}$ Ligation of GDNF to GFR $\alpha 1$ leads to the recruitment and activation of the receptor tyrosine kinase Ret, and to the consequent activation of several signaling cascades, including the mitogenactivated protein kinase pathway. ${ }^{2,11}$

GDNF is an important factor for the survival, regeneration and maintenance of DA-ergic midbrain neurons, ${ }^{1,2}$ and an increase in the GDNF levels has been suggested to be beneficial for Parkinson's disease ${ }^{2,12}$ and chronic stress. ${ }^{13}$ Moreover, activation of the GDNF pathway in the VTA has recently been suggested to negatively regulate the intake of drugs of abuse, including alcohol. ${ }^{14,15}$ Specifically for alcohol, activation of the GDNF signaling pathway in the VTA results in a fast (minutes), but also a very sustained (at least $48 \mathrm{~h}$ ), reduction in excessive, 'binge-like,' alcohol consumption in rats. ${ }^{16,17}$ The rapid actions of GDNF are mediated by the rapid stimulation of VTA DA-ergic neurons and the reversal of alcohol-induced DA deficiency in the nucleus accumbens (NAc) ${ }^{7,18}$ However, the long-lasting suppressive actions of GDNF on alcohol consumption are not likely to be mediated by the recombinant growth factor, which degrades within several hours. Therefore, the mechanism mediating these protracted effects is unknown.

Treatment of the DA-ergic-like SHSY5Y cell line with GDNF induces a long-lasting increase in the growth factor's own expression, which subsequently causes a sustained activation of its signaling pathway. ${ }^{19}$ Specifically, a short exposure of SHSY5Y cells with recombinant GDNF (rGDNF) leads to a prolonged increase in the levels of the growth factor, and to the consequent long-lasting activation of Ret and the Ret-mediated activation of the mitogen-activated protein kinase extracellular-regulated protein kinase $1 / 2$ (ERK1/2). ${ }^{19}$

\footnotetext{
${ }^{1}$ The Ernest Gallo Research Center, University of California, San Francisco, Emeryville, CA, USA; ${ }^{2}$ Department of Neurology, University of California, San Francisco, San Francisco, CA, USA and ${ }^{3}$ The Graduate Program in Pharmaceutical Sciences and Pharmacogenomics, University of California, San Francisco, San Francisco, CA, USA

Correspondence: Professor D Ron, Department of Neurology, The Ernest Gallo Research Center, University of California, San Francisco, 5858 Horton St., Suite 200 , Emeryville, San Francisco, CA 94608, USA.

E-mail: dron@gallo.ucsf.edu

Keywords: addiction; alcohol; GDNF; growth factors; ventral tegmental area

Received 1 Septemeber 2011; revised 21 October 2011; accepted 1 November 2011
} 
Here we show that the maintenance of the suppressive effects of a single infusion of rGDNF into the VTA on excessive alcohol consumption are mediated by the capacity of GDNF to positively and sustainably autoregulate its own mRNA and protein levels in an autocrine manner.

\section{Materials and methods}

A detailed description of the methods and procedures that appear below, as well as information about reagents, preparation of GDNF short hairpin RNA recombinant adenovirus, quantitative/semi-quantitative reverse transcription PCR, western blot analysis, drug administration, immunohistochemistry and histology can be found in the Supplementary Information.

Animals. Male Long-Evans rats (Harlan, Indianapolis, IN, USA); $400-450 \mathrm{~g}$ at the time of the surgery) were housed under a 12-h light/dark cycle (lights on at $0700 \mathrm{~h}$ ) with food and water available ad libitum. All animal procedures in this report were approved by the Gallo Center Institutional Animal Care and Use Committee and were conducted in agreement with the Guide for the Care and Use of Laboratory Animals, National Research Council, 1996.

\section{Intracranial infusion of rGDNF and adenovirus (AdV)- shGDNF}

Intra-VTA rGDNF infusion. Surgery, cannula implantation and infusion of rGDNF $(10 \mu \mathrm{g}$ per side in $1 \mu \mathrm{l})$ into the VTA were conducted as previously described. ${ }^{17}$ See Supplementary Information for detailed information.

Intra-VTA AdV-shGDNF infusion. Infusion of viruses (AdV-shGDNF or Adv-Scrambled (SCR), $1.3 \times 10^{8} \mathrm{TU} \mathrm{ml}^{-1}$ ) into the VTA was conducted similarly to our previous report. ${ }^{7}$ See Supplementary Information for detailed information.

Intermittent-access to $20 \%$ alcohol in the two-bottle choice drinking procedure. The intermittent access to alcohol procedure was performed as previously described. ${ }^{16,17}$ Specifically, animals were given $24 \mathrm{~h}$ of concurrent access to one bottle of $20 \%$ volume/volume alcohol in tap water and one bottle of water, starting at $1100 \mathrm{~h}$ on Monday, Wednesday and Friday, with 24 or $48 \mathrm{~h}$ of alcohol-deprivation periods in between the alcohol-drinking sessions. The water and alcohol bottles were weighed after $30 \mathrm{~min}, 4$ and $24 \mathrm{~h}$ of access. Surgery, virus infusion and microinjection procedures started after 21 alcohol access sessions, when rats maintained a stable baseline of alcohol consumption of $5.5-6 \mathrm{~g} \mathrm{~kg}^{-1}$ per $24 \mathrm{~h}$. See Supplementary Information for full details.
Statistical analysis. Data generated from reverse transcription-PCR experiments were analyzed using paired $t$-test or one- or two-way analysis of variance with/without repeated measures, as specified. Alcohol drinking experiments were conducted in a within-subjects design or mixed-model design. Alcohol preference was calculated as the percentage of alcohol solution consumed relative to total fluid intake (alcohol + water). Student Newman-Keuls posthoc analysis was used where indicated. See Supplementary Information for full details.

\section{Results}

GDNF induces its own sustained expression and produces a long-term activation of the ERK1/2 pathway in the VTA. As the GDNF receptors GFR $\alpha 1$ and Ret are highly expressed in the VTA, ${ }^{8-10,20,21}$ we tested whether GDNF activates a positive autoregulatory loop in vivo in this brain region. To do so, rGDNF $(10 \mu \mathrm{g})$ was infused into one side of the VTA and vehicle into the other side. The VTA was dissected 12, 24 or $48 \mathrm{~h}$ later and the expression levels of GDNF were measured. As shown in Figure 1a-c, GDNF mRNA levels were significantly higher in the GDNF-infused side at all three time points, suggesting that the activation of the GDNF-mediated signaling pathway in the VTA leads to a sustained increase in GDNF mRNA that lasts for at least $48 \mathrm{~h}$.

Next, we tested whether a single application of rGDNF in the VTA results in a long-lasting activation of the GDNFmediated signaling pathway. Rats were infused with the recombinant growth factor $(10 \mu \mathrm{g})$ into one side of the VTA and vehicle into the other side. Twenty-four hours later, the immunoreactivity of phosphorylated and thus activated ERK1/2 (pERK1/2) was measured. As shown in Figure 1d, remarkably higher levels of $p E R K 1 / 2$ were detected in the rGDNF-infused side, predominantly in DA-ergic (tyrosine hydroxylase-positive) neurons, as compared with the vehicle-infused side. These results suggest that a single infusion of rGDNF into the VTA leads to an increase in the level of GDNF and to a long-lasting activation of the GDNF-mediated ERK $1 / 2$ activation in the VTA.

GDNF-mediated upregulation of GDNF levels in the VTA requires de novo protein synthesis. The results above suggest that the long-lasting increase in GDNF levels in the VTA is due to the maintenance of a positive autoregulatory feedback loop in which GDNF upregulates its own expression. If this is the case, then the maintenance of this loop should depend on de novo protein synthesis. To test this possibility, the protein synthesis inhibitor cycloheximide

\footnotetext{
Figure 1 A single infusion of recombinant glial cell line-derived neurotrophic factor (rGDNF) into the ventral tegmental area (VTA) produces a sustained induction of GDNF expression and phosphorylation of extracellular-regulated protein kinase 1/2 (ERK1/2). rGDNF (10 $\mu \mathrm{g}$, unilateral) or vehicle was infused into the VTA of rats $12 \mathrm{~h}$ (a), $24 \mathrm{~h}$ (b) or $48 \mathrm{~h}$ (c) before the VTA dissection. GDNF and GAPDH mRNA levels were determined using semi-quantitative reverse transcription PCR. GAPDH levels were measured to standardize sample loading. Data are expressed as mean \pm s.e.m. of GDNF/GAPDH expression ( $\$ s>3.50,{ }^{*} P<0.05 ; n=3-5$ ). (d) Shown is a dual-channel immunofluorescence for phospho-ERK1/2 (p-ERK1/2, red), tyrosine hydroxylase (TH, a marker for dopaminergic neurons, green), and overlay (yellow). Left panel: images depict ERK1/2 phosphorylation in the midbrain $24 \mathrm{~h}$ after rGDNF (left brain side) or PBS (right side) infusion into the VTA. Images are representative of results from four rats (six sections per rat), scale bar: $500 \mu \mathrm{m}$. Right panel: magnified image of the VTA infused with rGDNF, as marked in a white square in Figure 2a. The arrowheads point to cells immunostained for both $\mathrm{p}$-ERK1/2 and TH, scale bar: $50 \mu \mathrm{m}$.
} 
$\left(\mathrm{CHX} ; 2 \mathrm{mg} \mathrm{kg}^{-1} \text {, i.p. }\right)^{22,23}$ or vehicle was administered to rats $50 \mathrm{~min}$ before and $3 \mathrm{~h}$ after a unilateral intra-VTA rGDNF infusion, and the VTA was dissected 24 or $48 \mathrm{~h}$ after rGDNF infusion. $\mathrm{CHX}$ was administered twice at this interval to assure efficient inhibition of protein synthesis of at least $6-8 \mathrm{~h}^{24}$ As depicted in Figure $2 \mathrm{a}$, in control rats, we observed a robust increase in GDNF mRNA after $48 \mathrm{~h}$ in the side of the VTA that was infused with rGDNF, as a

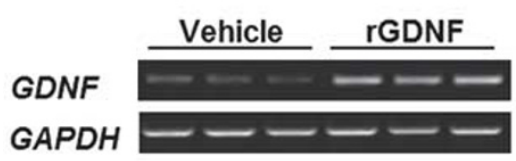

b

24 hrs

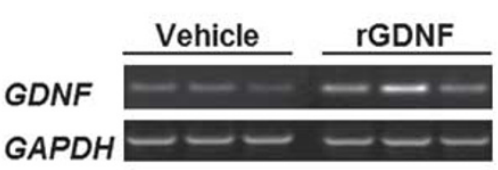

C

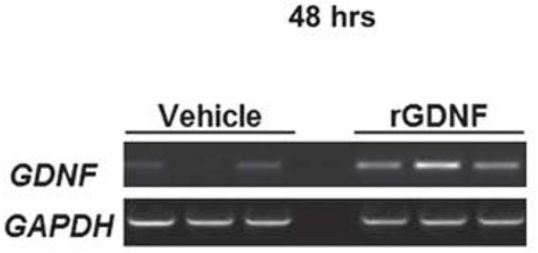

d

pERK1/2

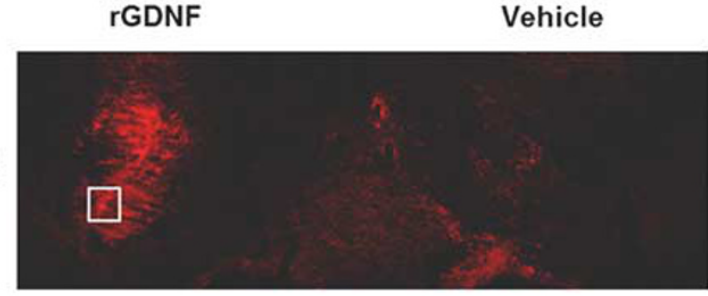

TH
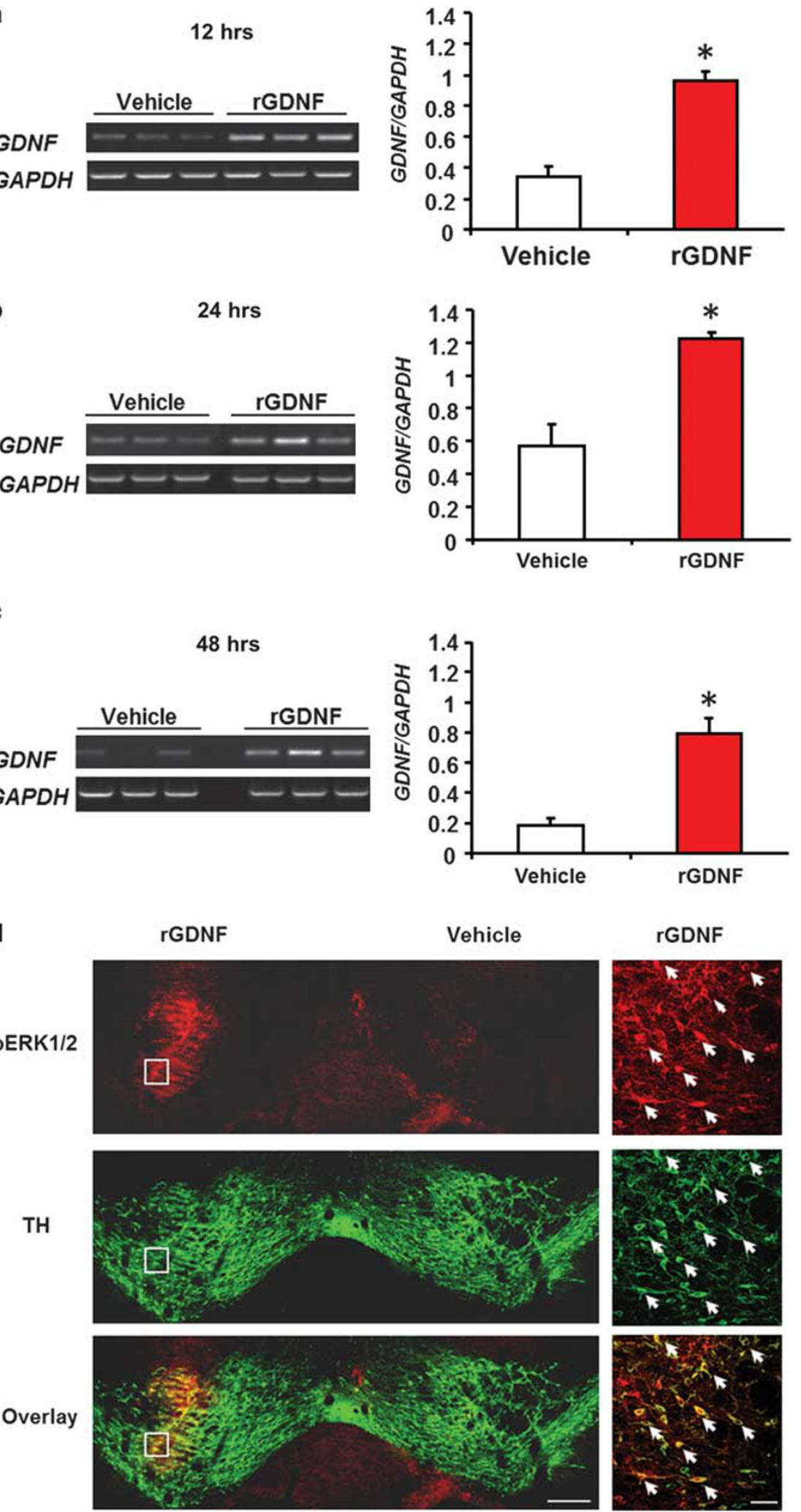


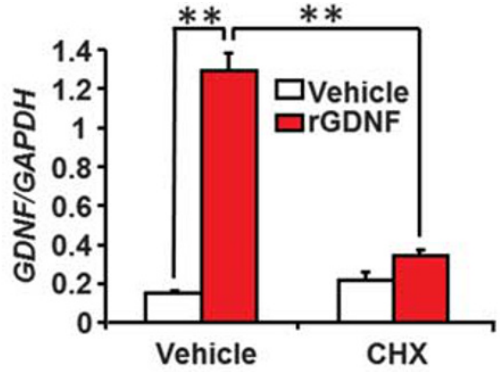

b
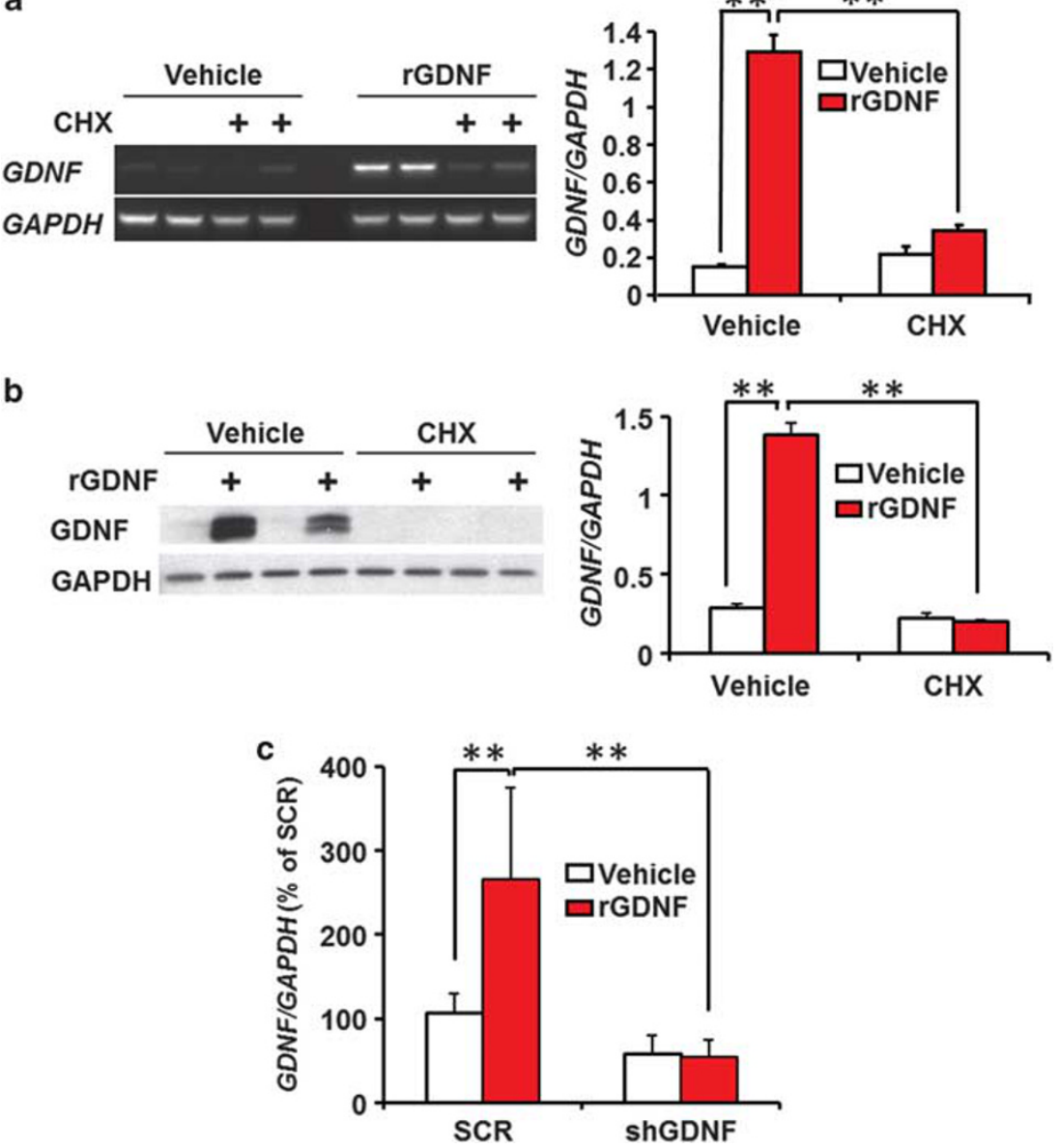

Figure 2 Recombinant glial cell line-derived neurotrophic factor (rGDNF)-mediated upregulation of GDNF mRNA and protein requires de novo protein translation. rGDNF $\left(10 \mu \mathrm{g}\right.$, unilateral) or vehicle was infused into the ventral tegmental area (VTA) of rats $48 \mathrm{~h}$ (a) or $24 \mathrm{~h}$ (b) before VTA dissection. Cycloheximide (CHX; $2 \mathrm{mg} \mathrm{kg}{ }^{-1}$, i.p.) was administered 50 min before and $3 \mathrm{~h}$ after rGDNF infusion. $\mathrm{CHX}$ was administered twice at these intervals to provide efficient inhibition of protein synthesis for at least 6-8 $\mathrm{h}^{24}$ (a) GDNF and GAPDH mRNA levels were determined using semi-quantitative reverse transcription PCR. Data are expressed as mean \pm s.e.m. of GDNF/GAPDH levels (main effects of $\mathrm{CHX}$ pretreatment $(\mathrm{F}(1,6)=67.03 ; P<0.0002)$ and $\mathrm{rGDNF}$ infusion $(\mathrm{F}(1,6)=156.88 ; P<0.0001$ ), and a significant interaction $(F(1,6)=101.09 ; P<0.0001)$. Post-hoc comparison: a significant difference between the rGDNF- and the vehicle-infused sides in rats that were pretreated with vehicle, but not in $\mathrm{CHX}$-pretreated rats; $\left.{ }^{* \star} P<0.001 ; n=4\right)$. (b) GDNF and GAPDH protein levels were determined using western blot analysis. Data are expressed as mean \pm s.e.m. of GDNF/GAPDH levels (main effects of $\mathrm{CHX}$ pretreatment $(F(1,6)=171.01 ; P<0.0001)$ and $\operatorname{rGDNF}$ infusion $(F(1,6)=281.47 ; P<0.0001)$, and a significant interaction $(F(1,6)=279.17 ; P<0.0001)$. Post-hoc comparison: a significant difference between the rGDNF- and the vehicle-infused sides in rats that were pretreated with vehicle, but not in $\mathrm{CHX}$-pretreated rats; ${ }^{*} P<0.001 ; n=4$ ). (c) Adenovirus shGDNF (AdV-shGDNF) or AdV-shSCR (1.2 $\mu \mathrm{l}$ per side, $1.3 \times 10^{8} \mathrm{TU} \mathrm{ml}^{-1}$ ) was infused into the VTA of rats. rGDNF was infused into the VTA 14 days after virus infection. The VTA was dissected $24 \mathrm{~h}$ later and GDNF mRNA levels were quantified by real-time reverse transcription PCR. Data are expressed as mean \pm s.e.m. of GDNF/GAPDH expression (main effects of viral infection $(F(1,12)=61.19, P<0.0001)$ and rGDNF infusion $(F(1,12)=16.57, P<0.002)$, and a significant interaction $(F(1,12)=18.37, P<0.002)$; post-hoc comparisons: a significant difference between rGDNF- and vehicle-infused VTA in the AdV-shSCR-infected rats ( $\left.{ }^{* \star} P<0.001\right)$, but not in the AdV-shGDNF-infected rats; $n=3-4)$

compared with the vehicle-infused VTA side. However, $\mathrm{CHX}$ pretreatment abolished these effects (Figure 2a). To confirm that a single infusion of rGDNF leads to a de novo synthesis of the endogenous protein, we measured GDNF levels in the VTA in the absence and presence of CHX. As shown in Figure $2 b$, infusion of the growth factor into the VTA produced a significant increase in the level of the protein, which was detected even $24 \mathrm{~h}$ later, and was abolished in response to $\mathrm{CHX}$ treatment. Together, these results suggest that inhibition of de novo protein synthesis disrupts the positive feedback loop of GDNF expression and signaling.

Next, we examined the consequences of downregulation of the GDNF transcript on the long-lasting increase in GDNF levels induced by a single administration of the recombinant polypeptide. To prevent the induction of GDNF mRNA, we used adenoviral-mediated gene delivery of short hairpin RNA, targeting GDNFmRNA (AdV-shGDNF), ${ }^{7}$ to specifically downregulate the level of the growth factor in the VTA of rats. This virus also expresses GFP, which was used to detect the infection locus. As shown in Supplementary Figure 1, AdVshGDNF infected DA-ergic (tyrosine hydroxylase-positive) neurons in the VTA and induced $\sim 40 \%$ decrease in GDNF mRNA expression in this brain region (day 14). Next, we unilaterally infused rGDNF $(10 \mu \mathrm{g})$ and vehicle into the VTA of rats that were infected with AdV-shGDNF or AdV-shSCR control in the VTA, 14 days earlier. We then examined the levels of GDNF $24 \mathrm{~h}$ after the infusion of the recombinant 
growth factor, and found that AdV-shGDNF-mediated downregulation of the endogenous gene is sufficient to disrupt the positive autoregulatory loop initiated by exogenous rGDNF (Figure 2c).

Inhibition of protein synthesis prevents the long-lasting, but not the acute, effects of GDNF on alcohol consumption. Next, we tested whether the activation of the positive autoregulatory feedback loop of GDNF signaling in the VTA underlies the ability of the growth factor to induce a longlasting decrease in alcohol intake. To do so, rats were trained in an intermittent access to $20 \%$ alcohol, two-bottle choice procedure for 7 weeks to obtain a baseline level of alcohol intake of $5.5-6 \mathrm{~g} \mathrm{~kg}^{-1}$ per $24 \mathrm{~h}$. This procedure generates a blood alcohol concentration of $80.9 \pm 7.2 \mathrm{mg} \%,{ }^{16}$ which corresponds to the blood alcohol concentration values that are defined by the National Institute on Alcohol Abuse and Alcoholism as binge drinking in humans. ${ }^{25}$ We then inhibited protein synthesis by administering $\mathrm{CHX}\left(2 \mathrm{mg} \mathrm{kg}^{-1}\right.$, i.p.) or vehicle $1 \mathrm{~h}$ before and $3 \mathrm{~h}$ after the beginning of the drinking session, and rGDNF or vehicle was bilaterally infused into the VTA $10 \mathrm{~min}$ before the beginning of the drinking session (Figure 3a). The immediate effects of GDNF on alcohol drinking were assessed by measuring alcohol consumption during the first $30 \mathrm{~min}$ of the drinking session, whereas the long-lasting effects were determined by measuring consumption during the period of $4-24 \mathrm{~h}$ after the beginning of the drinking session.

As previously reported, ${ }^{16}$ a single infusion of rGDNF into the VTA produced both a rapid $(30 \mathrm{~min})$ and a long-lasting $(4-24 \mathrm{~h})$ reduction in alcohol intake (Figure $3 \mathrm{~b}$ and Supple- mentary Figure 2A). CHX on its own did not alter the intake of alcohol (Figure $3 \mathrm{~b}$ and $\mathrm{c}$ ) or total fluid (Supplementary Figure 2C), and had no effect on the GDNF-mediated suppression of alcohol consumption during the first $30 \mathrm{~min}$ (Figure $3 b$ ). In contrast, CHX pretreatment blocked the longlasting (4-24 h) actions of GDNF on alcohol intake (Figure 3c), as well as alcohol preference (Supplementary Figure 2B). Together, these results suggest that the long-lasting, but not the immediate, effects of GDNF on alcohol consumption depend on de novo protein synthesis.

Knockdown of GDNF expression in the VTA prevents the long-lasting, but not the rapid, effects GDNF on alcohol consumption. If the positive feedback loop of GDNF in the VTA is the mechanism that underlies the long-lasting suppressive effects of GDNF on alcohol intake, then downregulation of GDNF in this region would prevent the long-lasting, but not the immediate, effects of GDNF on alcohol drinking. Rats were therefore trained in the intermittent access to $20 \%$ alcohol, two-bottle choice procedure for 7 weeks as described above. The VTA of the rats was then infected with AdV-shGDNF or AdV-shSCR. After a 3-day recovery period, rats were given three additional drinking sessions to re-establish baseline alcohol consumption. In the subsequent sessions, rGDNF or vehicle was infused into the VTA 10 min before the beginning of the drinking session, and the immediate and extended effects of GDNF on alcohol drinking were assessed. As shown in Figure 4, intra-VTA infusion of rGDNF rapidly suppressed alcohol consumption in the AdV-shSCR-infected rats (Figure 4a), and the inhibition of alcohol intake was also

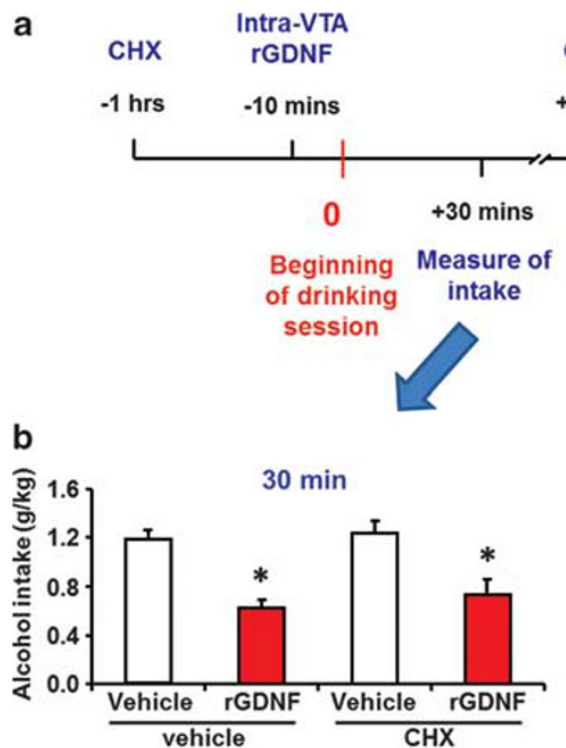

$\mathrm{CHX}$

$+3 \mathrm{hrs}$
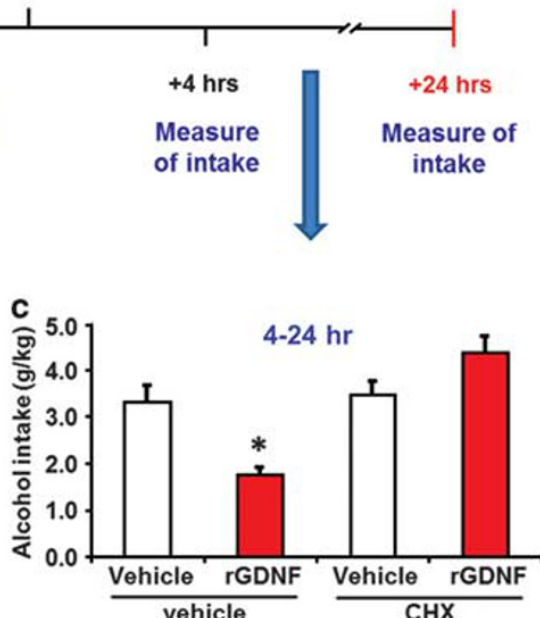

Figure 3 Inhibition of protein synthesis prevents the long-lasting, but not the acute, effects of recombinant glial cell line-derived neurotrophic factor (rGDNF) on alcohol consumption. Rats consumed a solution of $20 \%$ alcohol for 7 weeks. Cycloheximide (CHX; $2 \mathrm{mg} \mathrm{kg}^{-1}$, i.p.) was administered $1 \mathrm{~h}$ before and $3 \mathrm{~h}$ after the beginning of the alcohol-drinking session. rGDNF (10 $\mu \mathrm{g}$ per side) was infused into the ventral tegmental area (VTA) 10 min before the beginning of the drinking session. (a) Timeline of treatments and consumption measurements schedule. (a and $\mathbf{b})$ Data are expressed as mean \pm s.e.m. of alcohol intake in $\mathrm{g} \mathrm{kg}^{-1}\left({ }^{*} P<0.01\right.$ vs vehicle-vehicle; $n=12$ ). (b) Alcohol intake during the first $30 \mathrm{~min}$ of the drinking session (two-way repeated measurements analysis of variance (ANOVA): a main effect of rGDNF infusion $(\mathrm{F}(1,13)=34.79, P<0.0001)$, no effect of $\mathrm{CHX}$ pretreatment $(\mathrm{F}(1,13)=1.28, P=0.28)$, and no interaction $(\mathrm{F}(1,13)=0.07, P=0.79))$. (c) Alcohol intake during the 4-24-h time period after the beginning of the drinking session (two-way repeated measurements ANOVA: no effect of rGDNF infusion $(F(1,13)=2.19, P=0.16)$, a significant main effect of $\mathrm{CHX}$ pretreatment $(\mathrm{F}(1,13)=10.46, P<0.007)$, and a significant interaction $(F(1,13)=13.16, P<0.005)$. Post-hoc comparisons: a significant difference between rGDNF- and vehicle-infused rats in the vehicle control rats $(P<0.01)$, but not in rats that were pretreated with $\mathrm{CHX}(P=0.086))$. 
a

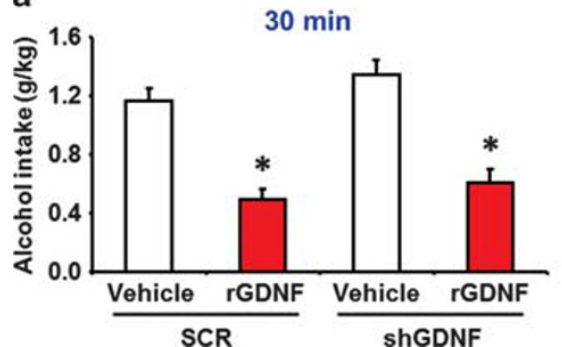

b

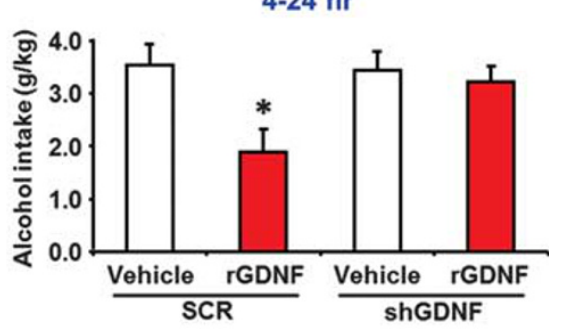

Figure 4 Downregulation of glial cell line-derived neurotrophic factor (GDNF) expression in the ventral tegmental area (VTA) prevents the sustained, but not the rapid, effects of recombinant GDNF (rGDNF) on alcohol consumption. Rats consumed a solution of $20 \%$ alcohol for 7 weeks. After the establishment of a baseline level of alcohol intake, VTA were infected with adenovirus (AdV)-shGDNF or control AdV-shSCR (SCR), after which the basal levels of alcohol intake were reestablished. Ten to 15 days after the infection, rGDNF ( $10 \mu \mathrm{g}$ per side) or vehicle was infused into the VTA $10 \mathrm{~min}$ before the drinking session onset. Data are expressed as mean \pm s.e.m. of alcohol intake in $\mathrm{g} \mathrm{kg}^{-1}{ }^{\star} P<0.01$ vs vehicle; $n=7$ ). (a) Alcohol intake during the first 30 min of the drinking session (main effects of rGDNF infusion $(\mathrm{F}(1,12)=40.55, P<0.0001)$ and of viral infection $(F(1,12)=4.89, P<0.05)$, but no interaction $(F(1,12)=0.10, P=0.75))$. (b) Alcohol intake during the $4-24 \mathrm{~h}$ time period after the beginning of the drinking session (mixed-model analysis of variance (ANOVA): a main effect of rGDNF infusion ( $F(1,12)=6.67, P<0.025)$, no effect of viral infection $(F(1,12)=2.53, P=0.13)$, and a marginally significant interaction $(\mathrm{F}(1,12)=3.88, P=0.072)$. Post-hoc comparisons: a difference between rGDNF- and vehicle-infused rats in the adenovirus (AdV)-shSCR infected control rats $(P<0.01)$, but not in rats that were infected with AdV-shGDNF $(P=0.67))$.

present in the 4-24-h consumption period (Figure 4b and see Supplementary Figure $3 \mathrm{~A}$ ). On its own, knockdown of GDNF in the VTA had no effect on alcohol intake (Figure 4a), preference (Supplementary Figure 3B) or on total fluid intake (Supplementary Figure 3C). However, the downregulation of GDNF expression level in the VTA prevented the longlasting inhibitory action of GDNF on alcohol consumption, but spared the immediate, rapid effects of the growth factor on alcohol intake (Figure $4 b$ ). These results suggest that the long-lasting effects of GDNF on alcohol consumption depend upon the initiation of GDNF-mediated transcription within the VTA, and the consequent translation of the growth factor.

\section{Discussion}

We report here that the administration of a single dose of rGDNF into the VTA results in a robust local induction of the growth factor's own expression and protein synthesis, resulting in a long-lasting activation of ERK1/2. We further show that the long-lasting elevation in GDNF levels depends upon de novo translation of this growth factor. These results suggest that a single administration of rGDNF into the VTA activates a positive autoregulatory feedback loop in which the activation of the GDNF-mediated signaling pathway in the VTA controls the expression of the growth factor (model, Figure 5). Importantly, we show that a single treatment with rGDNF produces a very long-lasting suppression of alcohol drinking, which is maintained/sustained by the ability of GDNF to positively autoregulate its own endogenous levels in the VTA.

Positive autoregulation of GDNF mediates the longlasting suppression of alcohol consumption. We previously found that activation of the GDNF pathway within the VTA rapidly suppresses excessive alcohol consumption. ${ }^{16,17}$ The rapid suppressive effects of GDNF on alcohol consumption are mediated directly by the exogenous rGDNF protein infused into the VTA, as they were independent of the inhibition of GDNF translation. These results, together with previous studies, ${ }^{7,18}$ imply that the immediate effects of intra-VTA rGDNF infusion on alcohol drinking are mediated by the exogenous rGDNF via a nongenomic mechanism.

We also found that the suppressive actions of rGDNF on alcohol are very long-lasting and are maintained for at least $48 \mathrm{~h},{ }^{16,17}$ and here we provide data to suggest that the GDNFmediated positive autoregulatory loop underlies the prolonged suppressive effects of GDNF on alcohol consumption. Specifically, we report that the long-lasting effects of a single intra-VTA infusion of rGDNF on alcohol consumption are prevented by the inhibition of protein synthesis, as well as by a focal knockdown of the GDNF transcript in the VTA. Thus, these prolonged effects depend upon the transcription and translation of the endogenous polypeptide. It is possible, therefore, that as long as the high levels of GDNF in the VTA are maintained, alcohol seeking and consumption are suppressed.

Knockdown of GDNF in the VTA prevented the long-lasting suppression of alcohol intake by rGDNF; however, the consumption of alcohol was unaffected by the downregulation of the growth factor in control animals that did not receive rGDNF. This result is puzzling, as activation of the GDNF signaling in this brain region leads to suppression of alcohol intake, ${ }^{16,17,26}$ and therefore, downregulation of the GDNF message is expected to enhance alcohol consumption. It should be borne in mind, however, that the basal levels of GDNF in the VTA are very low, ${ }^{10,27}$ and therefore, further downregulation of GDNF might not have significant behavioral outcomes. Alternatively, it is plausible that the negative result is due to a ceiling effect of alcohol intake, which could not be further increased upon knockdown of the growth factor.

GDNF positively autoregulates its own levels in vivo. We found that intra-VTA infusion of rGDNF results in a long-lasting upregulation of mRNA and protein levels of the growth factor. Importantly, GDNF was not detected in $\mathrm{CHX}$-pretreated animals $24 \mathrm{~h}$ following the infusion of the recombinant polypeptide into the VTA. CHX inhibits the synthesis of de novo GDNF, but the drug cannot alter the level of the exogenous recombinant polypeptide. 
a

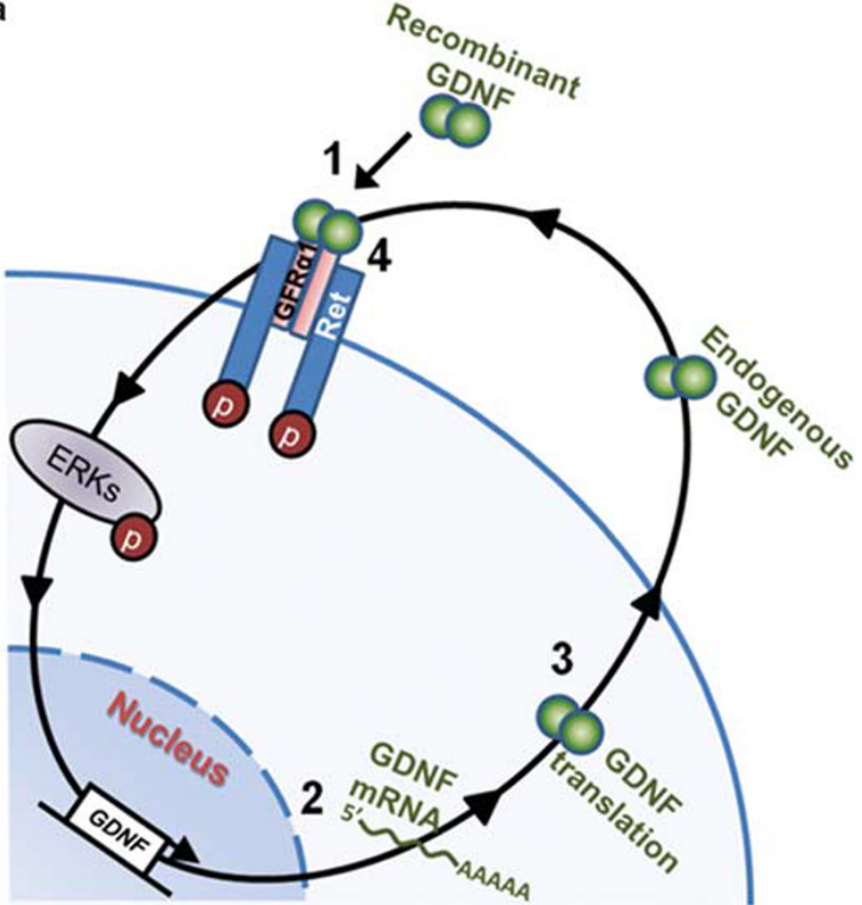

b

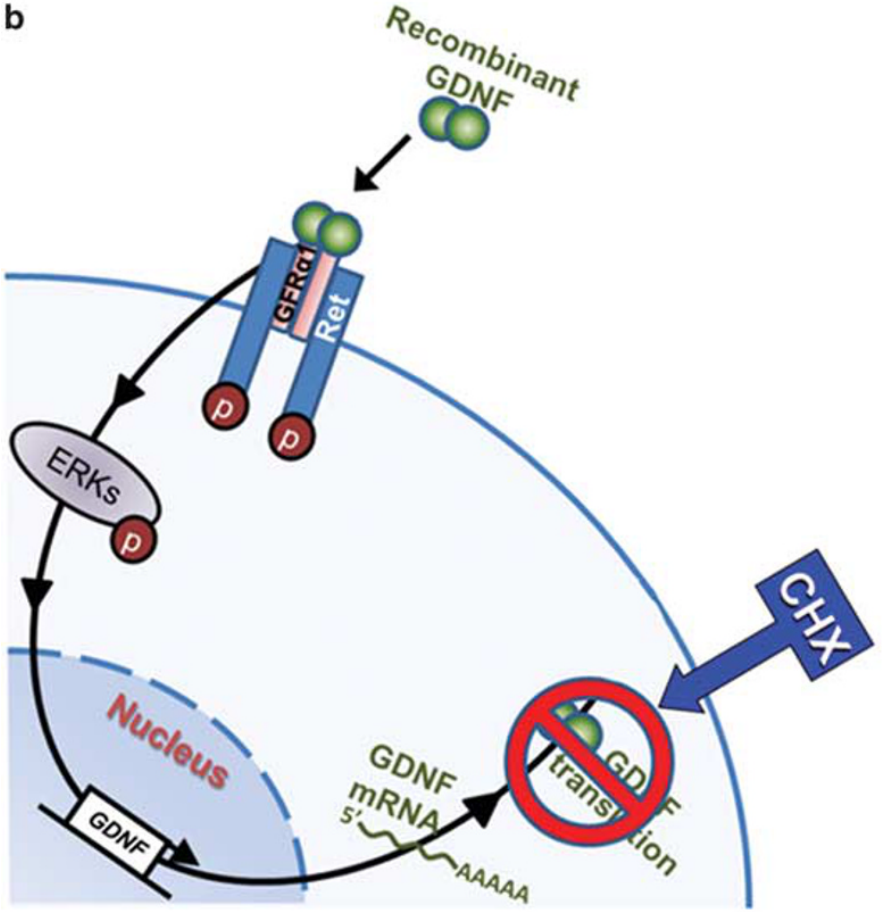

C

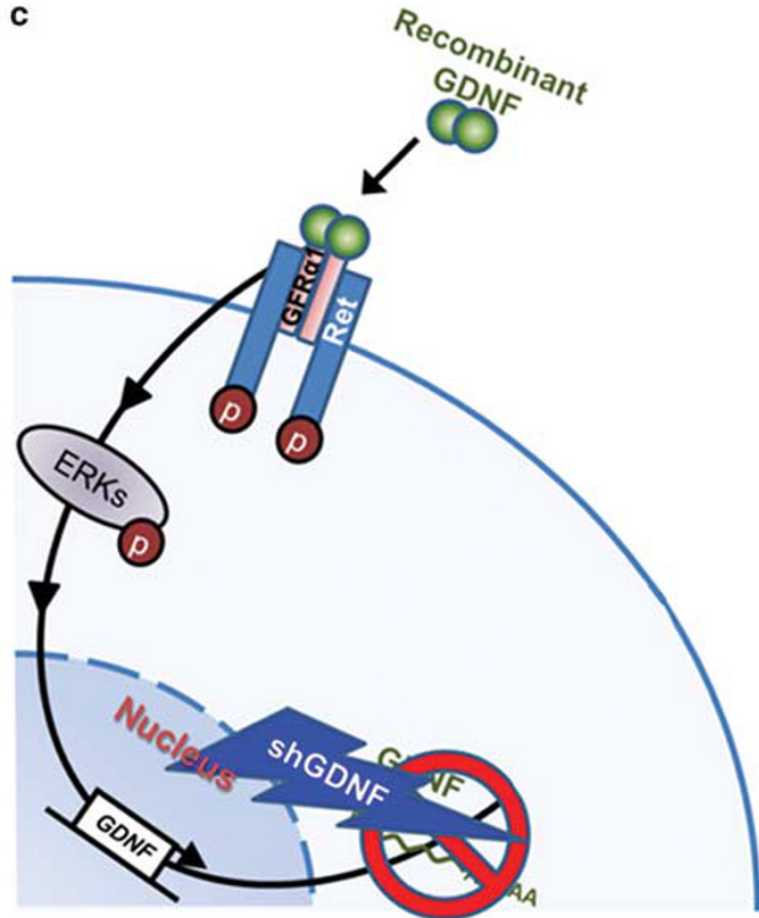

Figure 5 A schematic illustration of the glial cell line-derived neurotrophic factor (GDNF) autoregulatory loop within the ventral tegmental area (VTA). (a) Recombinant GDNF ( $r G D N F)$ binds to the GFR $\alpha 1$-Ret receptor complex in the VTA (1), which leads to the activation of Ret. Activation of the GDNF-mediated signaling pathway leads to the upregulation of the transcription of the GDNF gene (2). GDNF mRNA is translated to GDNF protein, and secreted (3). Secreted GDNF binds to its receptor complex (4), and activates its signaling pathway to maintain this positive feedback cycle. (b and $\mathbf{c}$ ) Inhibition of protein synthesis by cycloheximide (CHX) (b) or adenovirus (AdV)-shGDNFmediated downregulation of GDNF in the VTA (c) disrupts the autoregulatory feedback cycle by reducing the level of endogenously-produced GDNF, thus preventing the subsequent activation of the loop.

Thus, the exogenous rGDNF was degraded after a 24-h period, and therefore, the protein that was detected at this stage was the endogenously synthesized GDNF. Moreover, the long-lasting upregulation of GDNF expression that is induced by a single infusion of the recombinant polypeptide into the VTA is associated with a prolonged local activation of 
ERK1/2, suggesting that this pathway is part of the positive autoregulation cycle. Furthermore, as the increase in GDNF levels and the activation of Ret-mediated signaling pathway are detected within the same brain region, the autoregulatory loop is likely to be autocrine and not paracrine.

Positive autoregulation of expression and secretion of other neurotrophic factors has been demonstrated previously, albeit only in in vitro cell culture systems. For example, brain-derived neurotrophic factor positively regulates its own expression, ${ }^{28,29}$ secretion $^{30}$ and dendritic targeting of its own mRNA $^{31}$ in cortical and hippocampal cell cultures. Similarly, nerve growth factor and neurotrophin-4 were shown to increase their own expression in cortical cell cultures. ${ }^{29} \mathrm{We}$ provide here a unique demonstration that such a positive autoregulatory cycle occurs in vivo. Therefore, these findings may have significant implications for the function of other growth factors. Moreover, the downstream effector genes induced by the activation of the GDNF pathway are not well characterized, and our findings illuminate an in vivo pathway in which GDNF is its own effector gene.

At some point, however, the GDNF-mediated positive feedback cycle is likely to be terminated. One possibility is that long-term activation of GDNF signaling leads to a reduced functionality of its receptors by their internalization, degradation and/or ubiquitination. For example, Rap1GAP (a GTPase-activating protein for Rap1) binds to the Ret receptor, after the latter is activated by GDNF, and downregulates GDNF signaling. ${ }^{32}$ Similarly, dissociation of the CD2-associated protein and the E3-ligase Cbl-3 from the Ret receptor following the receptor's autophosphorylation leads to Ret degradation. ${ }^{33}$ Insufficient levels of Ret were shown to trigger internalization of GFR $\alpha 1$ receptors. ${ }^{34}$ Therefore, it is plausible that the positive and negative feedback loops of GDNF co-exist and compete with each other to regulate the levels of the growth factor. Specifically, whereas the levels of GDNF mRNA are upregulated by the positive feedback loop, the levels of the receptors are gradually reduced until they reach critical levels that attenuate GDNF signaling, to an extent that breaks the loop, and reduces the levels of the growth factor.

Physiological function of the GDNF-mediated positive autoregulatory loop. In the normal brain, within the mesolimbic and nigrostriatal systems, the GDNF receptors GFR $\alpha 1$ and Ret are highly expressed in the ventral midbrain, namely, the substantia nigra and the VTA, ${ }^{8-10,20,21}$ whereas the GDNF polypeptide itself is synthesized primarily in the $\mathrm{NAc} /$ striatum. ${ }^{3,4}$ Conversely, the VTA and substantia nigra normally express low basal levels of GDNF, ${ }^{10,27}$ and the $\mathrm{NAc/striatal-derived} \mathrm{GDNF}$ is retrogradely transported to the midbrain through DA-ergic projections. ${ }^{3,5-7,35,36}$

This mechanism might change when the normal conditions are disrupted. For example, damage to mesolimbic/nigrostriatal neurons leads to increased level of GDNF in the ventral midbrain. ${ }^{37-39}$ It is therefore plausible that under certain conditions such as brain insult, this positive autoregulatory mechanism has a role in the local amplification and prolongation of NAc-derived GDNF signaling. Once initiated, such a mechanism can provide a stable and faster supply of the growth factor, compared with the retrograde transportation of GDNF from the striatum/NAc, which requires several hours. ${ }^{7}$

Potential implications. We show here that GDNFmediated positive regulation of its own levels occurs in vivo in the VTA, a brain region where the actions of this growth factor were shown to have significant effects on the mesolimbic DA system. ${ }^{7,18}$ Our findings may have important implications to the understanding of the function of GDNF in the midbrain, as well as to the regulation of DA neurotransmission in the mesolimbic system. ${ }^{7,40}$ Furthermore, our results suggest pharmacological agents that increase GDNF levels ${ }^{26,27,41,42}$ can evoke a prolonged activation of the positive autoregulatory feedback loop, and can therefore sustainably suppress excessive alcohol intake, providing a long-lasting treatment strategy for alcohol use and abuse disorders, and possibly for other drugs of abuse. $^{14,15}$

Specifically for alcohol, we previously showed that infusion of rGDNF into the VTA reduces alcohol moderate and excessive operant self-administration, as well as relapse to alcohol drinking. ${ }^{17,18}$ More recently, we found that infusion of rGDNF into the VTA normalizes chronic alcohol-induced DA deficiency. ${ }^{19}$ Finally, we found that rGDNF prevents the acquisition as well as the expression of alcohol-conditioned place preference. ${ }^{18}$ Thus, this GDNF-mediated prolongationamplification mechanism might lead to a sustained suppression in the motivation to seek alcohol and/or in the rewarding properties of the latter.

In addition, it is highly likely that our findings are relevant to the effects of GDNF on self-administration of other drugs of abuse. ${ }^{14,15}$ For example, an activation of the GDNF pathway in the striatum decreases cocaine self-administration. ${ }^{43,44}$ Furthermore, a single intra-VTA rGDNF infusion after selfadministration was reported to increase cue-induced cocaine seeking on withdrawal days 3 and 10 ('incubation of cocaine craving'). ${ }^{45}$ Although it was previously suggested GDNF may cause differential effects depending on the timing of administration (i.e., before or after drug self-administration), ${ }^{15}$ it is also plausible that this long-term effect is also mediated by the capacity of a single rGDNF administration to maintain high levels of the growth factor in the VTA via the positive autoregulatory loop.

Importantly, increases in the growth factor have been proposed as a promising treatment strategy for Parkinson's disease, ${ }^{12,46-48}$ and reduced levels of GDNF have recently been implicated also in chronic stress. ${ }^{13}$ Therefore, our findings that exogenous activation of the GDNF pathway leads to a sustained upregulation of the growth factor in vivo may have beneficial implications for the development of treatments for Parkinson's disease and chronic stress.

\section{Conflict of interest}

The authors declare no conflict of interest.

Acknowledgements. This research was supported by funds provided by the National Institutes of Health-National Institute on Alcohol Abuse and Alcoholism (NIH-NIAAA) R01 AA014366 (DR), the State of California for Medical Research on 
Alcohol and Substance Abuse through the University of California San Francisco (DR), NIH-NIAAA Predoctoral Fellowship F31 AA017801 (SA), NIH Training Grant T32 GM007175 (SA), and Merck Laboratories Research Fellow award (SA). We thank Dr Sebastien Carnicella for the critical review of this manuscript.

1. Lin LF, Doherty DH, Lile JD, Bektesh S, Collins F. GDNF: a glial cell line-derived neurotrophic factor for midbrain dopaminergic neurons. Science 1993; 260: 1130-1132.

2. Airaksinen MS, Saarma M. The GDNF family: signalling, biological functions and therapeutic value. Nat Rev Neurosci 2002; 3: 383-394.

3. Barroso-Chinea P, Cruz-Muros I, Aymerich MS, Rodriguez-Diaz M, Afonso-Oramas D, Lanciego JL et al. Striatal expression of GDNF and differential vulnerability of midbrain dopaminergic cells. Eur J Neurosci 2005; 21: 1815-1827.

4. Pochon NA, Menoud A, Tseng JL, Zurn AD, Aebischer P. Neuronal GDNF expression in the adult rat nervous system identified by in situ hybridization. Eur J Neurosci 1997; 9: 463-471.

5. Tomac A, Widenfalk J, Lin LF, Kohno T, Ebendal T, Hoffer BJ et al. Retrograde axonal transport of glial cell line-derived neurotrophic factor in the adult nigrostriatal system suggests a trophic role in the adult. Proc Natl Acad Sci USA 1995; 92: 8274-8278.

6. Kordower JH, Emborg ME, Bloch J, Ma SY, Chu Y, Leventhal L et al. Neurodegeneration prevented by lentiviral vector delivery of GDNF in primate models of Parkinson's disease. Science 2000; 290: 767-773

7. Wang J, Carnicella S, Ahmadiantehrani S, He DY, Barak S, Kharazia V et al. Nucleus accumbens-derived glial cell line-derived neurotrophic factor is a retrograde enhancer of dopaminergic tone in the mesocorticolimbic system. $J$ Neurosci 2010; 30 14502-14512.

8. Burazin TC, Gundlach AL. Localization of GDNF/neurturin receptor (c-ret, GFRalpha-1 and alpha-2) mRNAs in postnatal rat brain: differential regional and temporal expression in hippocampus, cortex and cerebellum. Brain Res Mol Brain Res 1999; 73: 151-171.

9. Matsuo A, Nakamura S, Akiguchi I. Immunohistochemical localization of glial cell linederived neurotrophic factor family receptor alpha-1 in the rat brain: confirmation of expression in various neuronal systems. Brain Res 2000; 859: 57-71.

10. Trupp M, Belluardo N, Funakoshi H, Ibanez CF. Complementary and overlapping expression of glial cell line-derived neurotrophic factor (GDNF), c-ret proto-oncogene, and GDNF receptor-alpha indicates multiple mechanisms of trophic actions in the adult rat CNS. J Neurosci 1997; 17: 3554-3567.

11. Sariola H, Saarma M. Novel functions and signalling pathways for GDNF. J Cell Sci 2003; 116(Part 19): 3855-3862.

12. Sherer TB, Fiske BK, Svendsen CN, Lang AE, Langston JW. Crossroads in GDNF therapy for Parkinson's disease. Mov Disord 2006; 21: 136-141.

13. Uchida S, Hara K, Kobayashi A, Otsuki K, Yamagata H, Hobara T et al. Epigenetic status of Gdnf in the ventral striatum determines susceptibility and adaptation to daily stressful events. Neuron 2011; 69: 359-372.

14. Carnicella S, Ron D. GDNF-a potential target to treat addiction. Pharmacol Ther 2009; 122: 9-18.

15. Ghitza UE, Zhai H, Wu P, Airavaara M, Shaham Y, Lu L. Role of BDNF and GDNF in drug reward and relapse: A review. Neurosci Biobehav Rev 2009; 157-171.

16. Carnicella $S$, Amamoto R, Ron D. Excessive alcohol consumption is blocked by glial cell line-derived neurotrophic factor. Alcohol 2009; 43: 35-43.

17. Carnicella S, Kharazia V, Jeanblanc J, Janak PH, Ron D. GDNF is a fast-acting potent inhibitor of alcohol consumption and relapse. Proc Natl Acad Sci USA 2008; 105: 8114-8119.

18. Barak S, Carnicella S, Yowell QV, Ron D. Glial cell line-derived neurotrophic factor reverses alcohol-induced allostasis of the mesolimbic dopaminergic system: implications for alcohol reward and seeking. J Neurosci 2011; 31: 9885-9894.

19. He DY, Ron D. Autoregulation of glial cell line-derived neurotrophic factor expression: implications for the long-lasting actions of the anti-addiction drug, Ibogaine. FASEB J 2006; 20: $2420-2422$

20. Golden JP, Baloh RH, Kotzbauer PT, Lampe PA, Osborne PA, Milbrandt J et at. Expression of neurturin, GDNF, and their receptors in the adult mouse CNS. J Comp Neurol 1998; 398: 139-150

21. Glazner GW, Mu X, Springer JE. Localization of glial cell line-derived neurotrophic facto receptor alpha and c-ret mRNA in rat central nervous system. J Comp Neurol 1998; 391 : 42-49.

22. Obrig TG, Culp WJ, McKeehan WL, Hardesty B. The mechanism by which cycloheximide and related glutarimide antibiotics inhibit peptide synthesis on reticulocyte ribosomes. J Biol Chem 1971; 246: 174-181.

23. Siegel MR, Sisler HD. Inhibition of protein synthesis in vitro by cycloheximide. Nature 1963 200: 675-676.

24. Milekic MH, Brown SD, Castellini C, Alberini CM. Persistent disruption of an established morphine conditioned place preference. J Neurosci 2006; 26: 3010-3020.

25. National_Institute_on_Alcohol_Abuse_and_Alcoholism (NIAAA). Council approves definition of binge drinking. NIAAA Newsletter 2004; 3 .
26. Carnicella S, Ahmadiantehrani S, He DY, Nielsen CK, Bartlett SE, Janak PH et al. Cabergoline decreases alcohol drinking and seeking behaviors via glial cell line-derived neurotrophic factor. Biol Psychiatry 2009; 66: 146-153.

27. He DY, McGough NN, Ravindranathan A, Jeanblanc J, Logrip ML, Phamluong K et al. Glial cell line-derived neurotrophic factor mediates the desirable actions of the antiaddiction drug ibogaine against alcohol consumption. J Neurosci 2005; 25: 619-628.

28. Xiong H, Futamura T, Jourdi H, Zhou H, Takei N, Diverse-Pierluissi M et al. Neurotrophins induce BDNF expression through the glutamate receptor pathway in neocortical neurons. Neuropharmacology 2002; 42: 903-912.

29. Patz S, Wahle P. Neurotrophins induce short-term and long-term changes of cortical neurotrophin expression. Eur J Neurosci 2004; 20: 701-708.

30. Canossa M, Griesbeck O, Berninger B, Campana G, Kolbeck R, Thoenen H. Neurotrophin release by neurotrophins: implications for activity-dependent neuronal plasticity. Proc Natl Acad Sci USA 1997; 94: 13279-13286.

31. Righi M, Tongiorgi E, Cattaneo A. Brain-derived neurotrophic factor (BDNF) induces dendritic targeting of BDNF and tyrosine kinase $B$ mRNAs in hippocampal neurons through a phosphatidylinositol-3 kinase-dependent pathway. J Neurosci 2000; 20: 3165-3174.

32. Jiao L, Zhang Y, Hu C, Wang YG, Huang A, He C. Rap1GAP interacts with RET and suppresses GDNF-induced neurite outgrowth. Cell Res 2011; 21: 327-337.

33. Tsui CC, Pierchala BA. CD2AP and $\mathrm{Cbl}-3 / \mathrm{Cbl}-\mathrm{c}$ constitute a critical checkpoint in the regulation of ret signal transduction. $J$ Neurosci 2008; 28: 8789-8800.

34. Vieira P, Thomas-Crusells J, Vieira A. Internalization of glial cell-derived neurotrophic factor receptor GFR alpha 1 in the absence of the ret tyrosine kinase coreceptor. Cell Mol Neurobiol 2003; 23: 43-55

35. Ai Y, Markesbery W, Zhang Z, Grondin R, Elseberry D, Gerhardt GA et al. Intraputamenal infusion of GDNF in aged rhesus monkeys: distribution and dopaminergic effects. J Comp Neurol 2003; 461: 250-261.

36. Lapchak PA, Jiao S, Collins F, Miller PJ. Glial cell line-derived neurotrophic factor: distribution and pharmacology in the rat following a bolus intraventricular injection. Brain Res 1997; 747: 92-102.

37. Mandel S, Grunblatt E, Maor G, Youdim MB. Early and late gene changes in MPTP mice model of Parkinson's disease employing cDNA microarray. Neurochem Res 2002; 27: 1231-1243.

38. Yurek DM, Fletcher-Turner A. Temporal changes in the neurotrophic environment of the denervated striatum as determined by the survival and outgrowth of grafted fetal dopamine neurons. Brain Res 2002; 931: 126-134.

39. Grunblatt E, Mandel S, Maor G, Youdim MB. Gene expression analysis in N-methyl-4phenyl-1,2,3,6-tetrahydropyridine mice model of Parkinson's disease using cDNA microarray: effect of R-apomorphine. J Neurochem 2001; 78: 1-12.

40. Yang F, Feng L, Zheng F, Johnson SW, Du J, Shen L et al. GDNF acutely modulates excitability and A-type $\mathrm{K}(+)$ channels in midbrain dopaminergic neurons. Nat Neurosci 2001; 4: 1071-1078.

41. Carnicella S, He DY, Yowell QV, Glick SD, Ron D. Noribogaine, but not 18-MC, exhibits similar actions as ibogaine on GDNF expression and ethanol self-administration. Addict Biol 2010; 15: 424-433.

42. Saavedra A, Baltazar G, Duarte EP. Driving GDNF expression: the green and the red traffic lights. Prog Neurobiol 2008; 86: 186-215.

43. Green-Sadan T, Kinor N, Roth-Deri I, Geffen-Aricha R, Schindler CJ, Yadid G. Transplantation of glial cell line-derived neurotrophic factor-expressing cells into the striatum and nucleus accumbens attenuates acquisition of cocaine self-administration in rats. Eur J Neurosci 2003; 18: 2093-2098.

44. Green-Sadan T, Kuttner Y, Lublin-Tennenbaum T, Kinor N, Boguslavsky Y, Margel S et al. Glial cell line-derived neurotrophic factor-conjugated nanoparticles suppress acquisition of cocaine self-administration in rats. Exp Neurol 2005; 194: 97-105

45. Lu L, Wang X, Wu P, Xu C, Zhao M, Morales M et al. Role of ventral tegmental area glial cell line-derived neurotrophic factor in incubation of cocaine craving. Biol Psychiatry 2009; 66: $137-145$

46. Hong M, Mukhida K, Mendez I. GDNF therapy for Parkinson's disease. Expert Rev Neurother 2008: 8: 1125-1139.

47. Xia CF, Boado RJ, Zhang Y, Chu C, Pardridge WM. Intravenous glial-derived neurotrophic factor gene therapy of experimental Parkinson's disease with Trojan horse liposomes and a tyrosine hydroxylase promoter. J Gene Med 2008; 10: 306-315.

48. Manfredsson FP, Okun MS, Mandel RJ. Gene therapy for neurological disorders: challenges and future prospects for the use of growth factors for the treatment of Parkinson's disease. Curr Gene Ther 2009; 9: 375-388.

Translational Psychiatry is an open-access journal published by Nature Publishing Group. This work is licensed under the Creative Commons Attribution-Noncommercial-No Derivative Works 3.0 Unported License. To view a copy of this license, visit http://creativecommons.org/licenses/by-nc-nd/3.0/ 